\title{
Variability in the emergency department use of discretionary radiographs in children with common respiratory conditions: the mixed effect of access to pediatrician care
}

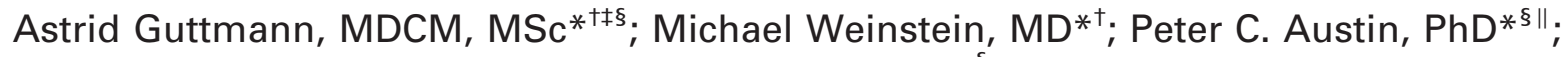 \\ Azim Bhamani, MSc*; Geoffrey Anderson, MD, $\mathrm{PhD}^{* \S}$
}

\section{ABSTRACT}

Objectives: The objective of this study was to investigate whether different staffing models are associated with variation in radiograph use for children seen for bronchiolitis, croup, and asthma and discharged home from emergency departments (EDs) in Ontario.

Methods: We surveyed all Ontario EDs regarding physician staffing models and use of clinical protocols. We used a population-based ED database to determine radiograph rates and patient characteristics. Regression techniques that controlled for patient factors and clustering within EDs were applied.

Results: From April 2004 to March 2006, 5,186, 10,408, and 35,150 children were discharged home from an ED with bronchiolitis, croup, and asthma, respectively. Radiograph rates were $42.7 \%$ for bronchiolitis, $10.1 \%$ for croup, and $25.9 \%$ for asthma. Over $50 \%$ of children were treated in EDs with nonpediatric front-line care but with consultant pediatricians available. Compared to children in these settings, those seen in EDs with front-line pediatric staff were less likely to have radiographs for all three conditions (adjusted odds ratios [ORs] 0.47 [95\% Cl 0.24-0.95], 0.47 [95\% Cl 0.27-0.82], 0.13 [95\% Cl 0.02-0.66] for bronchiolitis, croup, and asthma, respectively). Children in community hospitals with pediatricians were significantly more likely to have a radiograph if seen by a consultant pediatrician (OR 1.40, 95\% Cl 1.20-1.63 [bronchiolitis]; OR 2.76, 95\% Cl 2.16-3.53 [croup]; and OR 1.97, 95\% $\mathrm{Cl} 1.64-2.36$ [asthma]). We found no association between clinical protocol use and radiograph rates.

Conclusions: High rates of discretionary radiograph use exist for common respiratory problems of children seen across ED settings. Quality improvement efforts should be focused in this area, and radiograph use in EDs staffed by front-line pediatrics-trained staff could serve as an initial benchmark target for other institutions.

\section{RÉSUMÉ}

Objectifs: L'étude visait à examiner l'existence d'un lien entre différents modèles de dotation en personnel et les différences de recours à la radiographie chez les enfants souffrant de bronchiolite, de croup ou d'asthme, et ayant obtenu leur congé du service des urgences pour retourner à domicile, en Ontario.

Méthode: Une enquête a été menée dans tous les services d'urgence en Ontario en ce qui concerne les modèles de dotation en personnel médical et l'application de protocoles cliniques. Nous avons effectué des recherches dans une base de données sur les consultations aux services des urgences, fondée sur la population, afin de déterminer les taux de radiographies et les caractéristiques des patients. Des techniques de régression permettant de neutraliser les facteurs relatifs aux patients et les concentrations de cas dans les services des urgences ont été appliquées.

Résultats: D'avril 2004 à mars 2006, des enfants atteints de bronchiolite, de croup, ou d'asthme, au nombre de 5,186, 10,408 , et 35,150 , respectivement, ont obtenu leur congé du service des urgences pour retourner à domicile. Les taux de radiographies se répartissaient comme suit: $42.7 \%$ pour la bronchiolite, $10.1 \%$ pour le croup, et $25.9 \%$ pour l'asthme. Plus de $50 \%$ des enfants ont reçu des soins de première ligne non pédiatriques, mais en consultation avec des

See the related editorial/commentary in this issue on page 1.

From the *Division of Paediatric Medicine, The Hospital for Sick Children; †Department of Paediatrics, Faculty of Medicine, University of Toronto; flnstitute for Clinical Evaluative Sciences; §Department of Health Policy, Management and Evaluation, University of Toronto; and IIDalla Lana School of Public Health, University of Toronto, Toronto, ON.

Correspondence to: Dr. Astrid Guttmann, Institute for Clinical Evaluative Sciences, G Wing, Sunnybrook and Women's College Health Sciences Centre, 2075 Bayview Avenue, Toronto, ON M4N 3M5; astrid.guttmann@ices.on.ca.

This article has been peer reviewed. 
pédiatres disponibles. Comparativement aux enfants examinés dans ce contexte, ceux qui l'ont été par du personnel spécialisé en soins pédiatriques de première ligne, aux services des urgences, étaient moins susceptibles d'être soumis à des radiographies, et ce, pour les trois affections (risque relatif approché [RRA] rajusté 0.47 [IC à $95 \% 0.24-$ 0.95 ], 0.47 [IC à $95 \% 0.27-0.82$ ], et 0.13 [IC à $95 \% 0.02-0.66$ ] pour la bronchiolite, le croup, et l'asthme, respectivement). Les enfants examinés dans des hôpitaux communautaires dotés de pédiatres étaient passablement plus susceptibles d'être soumis à des radiographies s'ils étaient vus par des pédiatres consultants (RRA 1.40, IC à 95\% 1.20-1.63 [bronchiolite], RRA 2.76, IC à 95\% 2.16-3.53 [croup], et RRA 1.97, IC à 95\% 1.64-2.36 [asthme]). Nous n'avons pas trouvé de lien entre l'application de protocoles cliniques et les taux de radiographies.

Conclusions: II existe des taux élevés de radiographies discrétionnaires pour des troubles respiratoires fréquents chez les enfants dans tous les services d'urgence. Des efforts d'amélioration de la qualité s'imposeraient dans le domaine, et le recours aux radiographies dans les services d'urgence dotés de personnel formés en soins pédiatriques de première ligne devrait servir, au début, de cible de référence dans les autres établissements.

Keywords: asthma, bronchiolitis, croup, emergency care, quality, radiography
Emergency department (ED) care is an integral component of acute care for children. In both Canada and the United States, only a small number of hospitals with ED physicians are trained specifically in pediatrics and pediatric emergency medicine. ${ }^{1}$ The majority of emergency care for children is provided in EDs where pediatricians are available for consultation or with no pediatricians. ${ }^{2}$ In Canada, nonpediatric teaching hospitals are generally staffed by physicians trained in emergency medicine, whereas community hospitals have a mix of general practitioners and physicians with emergency medicine certification. Despite these fundamentally different physician staffing models, only a few studies have explored whether structural resource differences impact the nature and quality of care children receive. The results of these studies have varied, with some finding no significant difference in care between pediatric and general emergency medicine physicians $^{3,4}$ and others suggesting that front-line staff trained in pediatrics are more likely than general physicians to adhere to guideline-based care. ${ }^{5,6}$ All of these studies have been limited by being based on a limited number of sites or self-reporting, and none have investigated the impact of pediatricians available for consultation in general EDs. Furthermore, none of these studies have investigated whether the use of standardized protocols can overcome differences in staff training from the perspective of optimizing clinical processes and outcomes of care for children.

Respiratory illnesses such as bronchiolitis, croup, and asthma are among the top 10 diagnoses for visits made by children to Canadian and US EDs. ${ }^{7,8}$ In previous work, to systematically define performance measures for ED care of children, the rates of radiographs for these conditions were chosen as indicators of care efficiency, effectiveness, and safety. ${ }^{9}$ Routine use of radiographs for the diagnosis and management of these conditions is both unnecessary and harmful. ${ }^{10-12}$ Overuse of radiographs leads to increased cost and ED length of stay, as well as unnecessary radiation exposure and patient discomfort. More specific to children with bronchiolitis, radiographs have been shown to increase the inappropriate use of antibiotics in both the $\mathrm{ED}^{13}$ and inpatient ${ }^{14,15}$ settings. Based on hospital reporting, considerable siteto-site variation in the use of these radiographs exists in Ontario ${ }^{10}$ and in the United States. ${ }^{16}$

The objective of this study was to investigate whether different staffing models are associated with variation in radiograph use for children seen for bronchiolitis, croup, and asthma and discharged home from EDs in Ontario. We hypothesized that EDs with staff specifically trained in pediatric emergency medicine and those with access to pediatricians for consultation would have lower rates of radiograph use.

\section{METHODS}

\section{Overall design}

We conducted a retrospective cohort study of all children 3 to 24 months of age with bronchiolitis or croup and 1 to 19 years of age with asthma who were seen and discharged from an Ontario ED between April 1, 2004, and March 31, 2006. Ontario is Canada's largest province and has a population of 12 million persons. We linked data from a survey of Ontario EDs to a population-based administrative health database of ED visits to evaluate the effect of different physician staffing models on radiograph use. The data sets used 
were the Discharge Abstract Database for hospitalizations, the National Ambulatory Care Reporting System (NACRS) for ED visits, and physician billings from the Ontario Health Insurance Plan (OHIP). Chart reabstraction studies have shown main discharge diagnoses to be valid in the NACRS. ${ }^{17}$ These administrative health data for Ontario are available for research purposes at the Institute for Clinical Evaluative Sciences through an agreement with the Ontario Ministry of Health and Long-Term Care. Anonymized unique identifiers allowed linkages of records within the databases. The Research Ethics Board of Sunnybrook Hospital approved this study.

\section{Measures and data collection}

We surveyed the EDs of all 164 acute care hospitals in Ontario during early 2005 regarding training of frontline medical staff, availability of consultants, and use and content of guidelines and order sets for specific conditions. The details of the survey are available elsewhere. ${ }^{18}$

We used International Classification of Diseases version 10-CA final diagnosis codes from our ED data set to define our cohorts. In the case of multiple visits for the same diagnosis, we chose the first. We restricted our final cohort to children who were discharged home because they have less severe illness than admitted patients, making radiograph use more discretionary, and focused on care provided by ED rather than inpatient physicians. For children who were transferred to another ED for assessment or treatment, we included the index visit to the initial ED only.

Our primary outcome was radiograph use (chest radiograph for bronchiolitis and asthma, chest or lateral neck radiograph for croup). Work for the Ontario Hospital Report has validated against hospital records that the radiograph rates for these three conditions are accurate. ${ }^{19}$

We linked to previous ED visits and hospitalizations, including birth records, to describe relevant medical comorbidities (gestational age, chronic lung disease, complex congenital heart disease, congenital tracheoesophageal problems, history of croup) and used a validated population-based asthma registry (Ontario Asthma Surveillance Information System) ${ }^{20}$ to assess previous asthma diagnosis and a previous admission in the past 2 years as a measure of asthma severity. We used the Canadian Triage and Acuity
Scale score to control for patient acuity on presentation. This score has been shown to be reliable ${ }^{21}$ and valid. ${ }^{22}$ Postal code was used to capture the mean neighbourhood income quintile at the level of dissemination area (average population of 650) based on the 2001 Canadian census. ${ }^{23}$

We categorized hospitals into the following three groups based on the type of staffing available: 1) academic hospitals with pediatrics-trained ED physicians, 2) academic hospitals with emergency medicinetrained ED physicians, 3) community hospitals with consultant pediatricians available, and 4) other community hospitals. We used the NACRS to determine the overall annual volume of pediatric visits and excluded those with low volumes ( $\leq 10$ th percentile). We used physician billing data from OHIP to assess which children in community hospitals had a pediatric consultation in the ED.

\section{Statistical analyses}

A logistic regression model was fit for our primary analyses, allowing us to account for possible within-ED homogeneity in patient outcomes using generalized estimating equations. ${ }^{24}$ To control for variation in hospital admission threshold and any resulting difference in the severity of illness of discharged patients, we developed a condition-specific measure of each ED's propensity to admit using a logistic regression model with admission as the outcome to derive odds ratios (ORs) for patient factors from the entire sample. Using the coefficients of this model, we calculated a hospital rate of observed to expected admissions. Covariates in our final models included age, gender, and ED propensity to admit, as well as any additional variables that significantly altered the estimates for the hospital types.

To test whether any relationship found with radiograph use and being seen in EDs with pediatric consultants was borne out at the individual patient level, we did a subgroup analysis of children seen at community hospitals with pediatricians and investigated the relationship of receiving a radiograph with having had a pediatric consultation. Finally, secondary analyses were conducted to examine within-hospital correlation between radiograph rates for the different indications. For each condition, we determined the observed or predicted radiograph ratio for each hospital. The predicted number of radiographs was derived from a logistic regression model using patient factors only. A 
mixed effects model was used to regress the hospitalspecific ratios on variables denoting the diagnoses (asthma, croup, and bronchiolitis) in addition to other hospital-specific characteristics. The effect of each diagnosis was modeled using a random effect, allowing the effect of that diagnosis to vary across hospitals. The within-hospital correlation was derived from the variance-covariance matrix of the random effects.

\section{RESULTS}

\section{Hospital survey}

We received responses from all 164 EDs surveyed and excluded 13 low-volume EDs. Of the 15 academic hospitals, 2 were freestanding children's hospitals and 1 had a separate ED for children. These three EDs were the only ones in our sample with front-line pediatric staff; three of the other teaching EDs had pediatrician consultants. Within the community setting, most were staffed by family physicians and 41 hospitals had pediatric consultants. Only 1 ED reported having a guideline for bronchiolitis (so we did not analyze this effect), 19 reported using guidelines for patients with croup, and 58 reported using guidelines for asthma. Seventeen EDs reported having standard order sets for asthma.

\section{ED visits for bronchiolitis, croup, and asthma}

Table 1, Table 2, and Table 3 describe pediatric ED visits for bronchiolitis, croup, and asthma, respectively, by hospital type. Overall, these visits account for almost $6 \%$ of total ED visits by children, and just over $50 \%$ of these visits were made to community hospitals with pediatricians available for consultation. Of the total study population, 5,186 with bronchioliltis, 10,408 with croup, and 35,150 with asthma were discharged home. Among discharged children, hospital-specific radiograph rates with interquartile ranges were $42.7 \%$ (22.2-55.4), 10.1\% (0-12.8), and 25.9\% (10.5-29.8) for bronchiolitis, croup, and asthma, respectively.

\section{Association of patient factors with radiograph rates}

Table 4 shows the adjusted ORs of radiograph use in discharged patients by condition. After adjustment for triage level, none of the other comorbidities were associated with radiograph use for croup or bronchiolitis.
For asthma, younger age and no previous asthma diagnosis were associated with radiograph use. Socioeconomic status as measured by neighbourhood income was not associated with radiograph use for bronchiolitis or asthma.

\section{Association of hospital resources with radiograph rates}

The adjusted models across all three conditions indicated that patients seen at EDs with front-line pediatric staff were significantly less likely to receive a radiograph compared to children seen at community hospitals with pediatricians $(p<0.05$ for the clustered model). A similar trend was seen for children treated at community hospitals without pediatricians, although for asthma, this was not statistically significant. Overall findings were very consistent for bronchiolitis and croup. Compared to children seen in community hospitals with pediatricians, children seen in EDs with front-line pediatric physicians were approximately $63 \%$ less likely and those seen in community hospitals without pediatricians were $27 \%$ less likely to receive a radiograph. The effect of pediatric front-line staffing was most pronounced for children with asthma (adjusted OR 0.12, 95\% CI 0.02-0.62). No difference was found for children being seen in a nonpediatric teaching hospital compared to a community hospital with pediatricians.

No association was found between being seen in an ED that used guidelines for croup or asthma or order sets for asthma and receiving a radiograph. Overall pediatric patient volume was not found to be associated with radiograph use except in asthma, where a very small increased risk was seen with increasing volume. A subgroup analysis of community hospitals with pediatricians found that at an individual level, after controlling for age, gender, and triage, children were significantly more likely to have a radiograph (OR 1.40, 95\% CI 1.20-1.63 [bronchiolitis]; OR 2.76, 95\% CI 2.16-3.53 [croup]; and OR 1.97, 95\% CI 1.64-2.36 [asthma]) if seen by a consultant pediatrician. There was a moderate correlation of radiograph use within EDs: 0.49 (bronchiolitis and asthma), 0.39 (croup and asthma), and 0.31 (croup and bronchiolitis).

\section{DISCUSSION}

Our findings indicate that high rates of discretionary radiograph use exist for common respiratory problems of children seen across ED settings. Our analysis 


\begin{tabular}{|c|c|c|c|c|c|}
\hline \multirow[b]{2}{*}{ Characteristic } & \multicolumn{5}{|c|}{ Hospital type, $n(\%)$} \\
\hline & $\begin{array}{c}\text { Front-line } \\
\text { pediatric care } \\
(n=1,544)\end{array}$ & $\begin{array}{l}\text { Other academic } \\
\qquad(n=231)\end{array}$ & $\begin{array}{l}\text { Community with } \\
\text { pediatricians } \\
(n=3,681)\end{array}$ & $\begin{array}{l}\text { Community with } \\
\text { no pediatricians } \\
(n=1,352)\end{array}$ & Overall \\
\hline Male & $948(61.4)$ & $150(64.9)$ & $2,319(63.0)$ & $811(60.0)$ & $4,288(62.0)$ \\
\hline \multicolumn{6}{|l|}{ Age (mo) } \\
\hline $3-12$ & $1,126(73.0)$ & $153(66.2)$ & $2,425(65.9)$ & $857(63.4)$ & $4,561(67.0)$ \\
\hline $12-24$ & $418(27.0)$ & 78 (33.8) & $1,256(34.1)$ & 495 (36.6) & $2,247(33.0)$ \\
\hline \multicolumn{6}{|l|}{ Gestational age (wk) } \\
\hline$>36$ & $1,281(83.0)$ & $185(80.1)$ & $3,013(81.8)$ & $1,122(83.0)$ & $5,601(82.3)$ \\
\hline $32-36$ & $146(9.5)$ & $20(8.6)$ & $369(10.0)$ & $126(9.3)$ & $661(9.7)$ \\
\hline$<32$ & $39(2.5)$ & $9(3.9)$ & $116(3.2)$ & $33(2.4)$ & $197(2.9)$ \\
\hline No birth record & $78(5.0)$ & $17(7.4)$ & $183(5.0)$ & $71(5.1)$ & $349(5.1)$ \\
\hline \multicolumn{6}{|l|}{ History of chronic lung disease } \\
\hline No & $1,457(94.4)$ & $213(92.2)$ & $3,477(94.5)$ & $1,273(94.1)$ & $6,420(94.3)$ \\
\hline Yes & $9(0.6)$ & $1(0.5)$ & $21(0.6)$ & $8(0.6)$ & $39(0.6)$ \\
\hline No birth record & $78(5.0)$ & $17(7.3)$ & $183(4.9)$ & $71(5.3)$ & $349(5.1)$ \\
\hline \multicolumn{6}{|l|}{ Complex congenital heart disease } \\
\hline No & 1,479 (95.8) & $221(95.5)$ & 3,565 (96.9) & $1321(97.7)$ & $6,586(96.7)$ \\
\hline Yes & $65(4.2)$ & $10(4.5)$ & $116(3.1)$ & $31(2.3)$ & $222(3.3)$ \\
\hline \multicolumn{6}{|l|}{ Neighbourhood income quintile } \\
\hline Missing & $3(0.2)$ & $1(0.5)$ & $39(1.1)$ & $30(2.2)$ & $73(1.1)$ \\
\hline 1 (lowest) & $464(30.0)$ & $72(31.2)$ & $808(22.0)$ & $311(23.0)$ & $1,655(24.3)$ \\
\hline 2 & $295(19.1)$ & $42(18.1)$ & $725(19.7)$ & $280(20.7)$ & $1,342(19.7)$ \\
\hline 3 & $245(15.9)$ & $46(19.9)$ & 723 (19.6) & $278(20.6)$ & $1,292(19.0)$ \\
\hline 4 & $264(17.1)$ & $37(16.0)$ & 807 (21.9) & $240(17.8)$ & $1,348(19.8)$ \\
\hline 5 (highest) & $273(17.7)$ & $33(14.3)$ & $579(15.7)$ & $213(15.7)$ & $1,098(16.1)$ \\
\hline \multicolumn{6}{|l|}{ CTAS score } \\
\hline 1-2 (resuscitation, emergent) & 259 (16.8) & 85 (36.8) & $1,417(38.5)$ & $178(13.2)$ & $1,939(28.5)$ \\
\hline 3 (urgent) & $1,088(70.4)$ & $113(48.9)$ & $1,931(52.4)$ & $646(47.8)$ & $3,778(55.5)$ \\
\hline 4-5 (semiurgent, nonurgent) & 197 (12.8) & $33(14.3)$ & $333(9.1)$ & $528(39.0)$ & $1,091(16.0)$ \\
\hline \multicolumn{6}{|l|}{ Disposition } \\
\hline Admitted & $162(10.5)$ & 76 (32.9) & $1,156(31.4)$ & 228 (16.9) & $1,622(23.8)$ \\
\hline Discharged & $1,382(89.5)$ & $155(67.1)$ & $2,525(68.6)$ & $1,124(83.1)$ & $5,186(76.2)$ \\
\hline Chest radiograph (admitted patients) & $122(75.3)$ & $53(69.7)$ & 888 (76.8) & $142(62.2)$ & $1,205(74.3)$ \\
\hline $\begin{array}{l}\text { Chest radiograph (discharged } \\
\text { patients) }\end{array}$ & $404(29.2)$ & $86(55.5)$ & $1,291(51.1)$ & $432(38.4)$ & $2,213(42.7)$ \\
\hline Overall radiograph rate & $526(34.1)$ & $139(60.2)$ & $2,179(59.2)$ & $574(42.5)$ & $3,418(50.2)$ \\
\hline
\end{tabular}

controlled for patient comorbidity and other ED resources, such as clinical protocols, that could explain this finding. A recent study suggests that such discretionary radiograph use is even higher in US EDs. ${ }^{16}$ Across all three conditions, we found a consistent relationship involving less radiograph use in EDs with front-line pediatric staff and, contrary to our hypothesis, increased radiograph use in those with pediatrician consultant availability. The former finding is in keeping with other studies documenting higher compliance with pediatric guideline care by ED physicians trained in pediatrics. ${ }^{5,6}$ One previous study found a sixfold increase in adjusted radiograph rates for children with croup seen in EDs staffed by general versus pediatric emergency medicine physicians. ${ }^{25}$ However, to our knowledge, ours is the first study to find an increase in radiograph use associated with the availability of pediatric consultant staff. Our data cannot explore at what point in care children who are seen by consultants receive radiographs or which physician ordered it. It is possible that a culture of "do everything before you call the consultant" could 


\begin{tabular}{|c|c|c|c|c|c|}
\hline \multirow[b]{2}{*}{ Characteristic } & \multicolumn{5}{|c|}{ Hospital type, $n(\%)$} \\
\hline & $\begin{array}{c}\text { Front-line } \\
\text { pediatric care } \\
(n=1,851)\end{array}$ & $\begin{array}{l}\text { Other academic } \\
\qquad(n=458)\end{array}$ & $\begin{array}{l}\text { Community with } \\
\text { pediatricians } \\
(n=5,611)\end{array}$ & $\begin{array}{l}\text { Community with } \\
\text { no pediatricians } \\
(n=3,131)\end{array}$ & Overall \\
\hline Male & $1,166(63.0)$ & $308(67.2)$ & $3,563(63.5)$ & $1,983(63.3)$ & $7,020(63.5)$ \\
\hline \multicolumn{6}{|l|}{ Age (mo) } \\
\hline 3-12 & 700 (37.8) & $171(37.3)$ & 2,007 (35.8) & 1,138 (36.3) & $4,016(36.3)$ \\
\hline $12-24$ & $1,151(62.2)$ & $287(62.7)$ & $3,604(64.2)$ & $1,993(63.7)$ & $7,035(63.7)$ \\
\hline \multicolumn{6}{|l|}{ Gestational age (wk) } \\
\hline$>36$ & $1,582(85.5)$ & $383(83.6)$ & 4,906 (87.4) & $2,726(87.1)$ & $9,597(86.8)$ \\
\hline $32-36$ & $153(8.3)$ & $34(7.4)$ & $356(6.4)$ & $233(7.4)$ & $776(7.0)$ \\
\hline$<32$ & $21(1.1)$ & $14(3.1)$ & $55(1.0)$ & $37(1.2)$ & $127(1.2)$ \\
\hline No birth record & $95(5.1)$ & $27(5.9)$ & $294(5.2)$ & $135(4.3)$ & $551(5.0)$ \\
\hline \multicolumn{6}{|l|}{ History of chronic lung disease } \\
\hline No & $1,752(94.7)$ & 430 (93.9) & $5,310(94.7)$ & 2,993 (95.6) & $10,485(94.9)$ \\
\hline Yes & $4(0.2)$ & $1(0.2)$ & $7(0.1)$ & $4(0.1)$ & $16(0.1)$ \\
\hline No birth record & $95(5.1)$ & $27(5.9)$ & $294(5.2)$ & $134(4.3)$ & $550(5.0)$ \\
\hline \multicolumn{6}{|l|}{$\begin{array}{l}\text { History of congenital } \\
\text { tracheoesophageal problems }\end{array}$} \\
\hline No & $1,851(100.0)$ & 457 (99.8) & $5,610(100.0)$ & $3,130(100.0)$ & 11,048 (99.9) \\
\hline Yes & $0(0.0)$ & $1(0.2)$ & $1(0.0)$ & $1(0.0)$ & $3(0.1)$ \\
\hline \multicolumn{6}{|l|}{ History of croup } \\
\hline No & $1,845(99.7)$ & $450(98.3)$ & $5,528(98.5)$ & $3,111(99.4)$ & $10,934(98.9)$ \\
\hline Yes & $6(0.3)$ & $8(0.7)$ & $83(1.5)$ & $20(0.6)$ & $117(1.1)$ \\
\hline \multicolumn{6}{|l|}{ Neighbourhood income quintile } \\
\hline Missing & $11(0.6)$ & $3(0.7)$ & $37(0.7)$ & $24(0.8)$ & $75(0.7)$ \\
\hline 1 (lowest) & $366(19.8)$ & $125(27.2)$ & $888(15.8)$ & $553(17.7)$ & $1,932(17.5)$ \\
\hline 2 & $342(18.5)$ & $97(21.2)$ & 981 (17.5) & $624(19.9)$ & $2,044(18.5)$ \\
\hline 3 & $337(18.2)$ & $81(17.7)$ & $1,052(18.8)$ & $691(22.1)$ & $2,161(19.6)$ \\
\hline 4 & $371(20.0)$ & $75(16.4)$ & $1,453(25.9)$ & $639(20.4)$ & $2,538(22.9)$ \\
\hline 5 (highest) & $424(22.9)$ & $77(16.8)$ & $1,200(21.3)$ & $600(19.1)$ & $2,301(20.8)$ \\
\hline \multicolumn{6}{|l|}{ CTAS score } \\
\hline 1-2 (resuscitation, emergent) & $168(9.1)$ & $114(24.9)$ & $1,763(31.4)$ & $313(10.0)$ & $2,358(21.3)$ \\
\hline 3 (urgent) & $1,135(61.3)$ & $259(56.6)$ & $3,264(58.2)$ & $1,525(48.7)$ & $6,183(56.0)$ \\
\hline 4-5 (semiurgent, nonurgent) & $548(29.6)$ & $85(18.5)$ & $584(10.4)$ & $1,293(41.3)$ & $2,510(22.7)$ \\
\hline \multicolumn{6}{|l|}{ Disposition } \\
\hline Admitted & $26(1.4)$ & $25(5.5)$ & $487(8.7)$ & $105(3.4)$ & $643(5.8)$ \\
\hline Discharged & $1,825(98.6)$ & $433(94.5)$ & $5,124(91.3)$ & $3,026(96.7)$ & $10,408(94.2)$ \\
\hline Chest radiograph (admitted patients) & $8(30.8)$ & $11(44.0)$ & $185(38.0)$ & $43(41.0)$ & $247(38.4)$ \\
\hline $\begin{array}{l}\text { Chest radiograph (discharged } \\
\text { patients) }\end{array}$ & $95(5.2)$ & 75 (17.3) & $643(12.6)$ & $235(7.8)$ & $1,048(10.1)$ \\
\hline Overall radiograph rate & $103(5.6)$ & $86(18.8)$ & $828(14.8)$ & 278 (8.9) & $1,295(11.7)$ \\
\hline
\end{tabular}

be responsible for our findings. Consultations may also reflect diagnostic uncertainty, which may lead to increased use of radiographs. Any efforts to reduce unnecessary radiograph use in hospitals with these arrangements will need to focus on the complex interaction of front-line and consulting staff, assess at which point of care radiographs are ordered, and determine whether any institutional practices might contribute to radiograph overuse.

Using the mean radiograph rate of front-line pediatric hospitals as a benchmark, strategies to reduce radiograph use in community hospitals with pediatric consultants could reduce the overall numbers of discharged children receiving radiographs by 1,570 in 


\begin{tabular}{|c|c|c|c|c|c|}
\hline \multirow[b]{2}{*}{ Characteristic } & \multicolumn{5}{|c|}{ Hospital type, $n(\%)$} \\
\hline & $\begin{array}{c}\text { Front-line } \\
\text { pediatric care } \\
(n=5,668)\end{array}$ & $\begin{array}{l}\text { Other academic } \\
\qquad(n=1,410)\end{array}$ & $\begin{array}{l}\text { Community with } \\
\text { pediatricians } \\
(n=19,485)\end{array}$ & $\begin{array}{l}\text { Community with } \\
\text { no pediatricians } \\
(n=12,899)\end{array}$ & Overall \\
\hline Male & $3,525(62.2)$ & $811(57.5)$ & $12,062(61.9)$ & $7,597(58.9)$ & $23,995(60.8)$ \\
\hline \multicolumn{6}{|l|}{ Age (yr) } \\
\hline $1-4$ & $3,124(55.1)$ & $444(31.5)$ & 7,962 (40.9) & $3,864(30.0)$ & $15,394(39.0)$ \\
\hline $5-9$ & $1,581(27.9)$ & $334(23.7)$ & $5,423(27.8)$ & $3,619(28.0)$ & $10,957(27.8)$ \\
\hline $10-14$ & 712 (12.6) & $255(18.1)$ & $3,363(17.3)$ & $2,815(21.8)$ & 7,145 (18.1) \\
\hline $15-19$ & $251(4.4)$ & $377(26.7)$ & $2,737(14.0)$ & $2,601(20.2)$ & $5,966(15.1)$ \\
\hline \multicolumn{6}{|l|}{ History of asthma } \\
\hline No & $1,841(32.5)$ & $404(28.7)$ & $4,841(24.8)$ & $3,838(29.8)$ & $10,924(27.7)$ \\
\hline Yes & $3,827(67.5)$ & $1,006(71.3)$ & $14,644(75.2)$ & $9,061(70.2)$ & $28,538(72.3)$ \\
\hline \multicolumn{6}{|l|}{$\begin{array}{l}\text { History of asthma admission in } 2 \mathrm{yr} \\
\text { pre-ED visit }\end{array}$} \\
\hline No & $5,285(93.2)$ & $1,307(92.7)$ & $17,450(89.6)$ & $12,174(94.4)$ & $36,216(91.8)$ \\
\hline Yes & $383(6.8)$ & $103(7.3)$ & 2,035 (10.4) & $725(5.6)$ & $3,246(8.2)$ \\
\hline \multicolumn{6}{|l|}{ Neighbourhood income quintile } \\
\hline Missing & $25(0.4)$ & $21(1.5)$ & $188(1.0)$ & $226(1.7)$ & $460(1.2)$ \\
\hline 1 (lowest) & $1,503(26.5)$ & $392(27.8)$ & $4,093(21.0)$ & $2,736(21.2)$ & $8,724(22.1)$ \\
\hline 2 & $1,132(20.1)$ & 309 (21.9) & $3,940(20.2)$ & $2,745(21.3)$ & $8,126(20.5)$ \\
\hline 3 & $971(17.1)$ & $258(18.3)$ & $3,791(19.5)$ & $2,719(21.1)$ & 7,739 (19.6) \\
\hline 4 & $1,032(18.2)$ & $233(16.5)$ & $4,192(21.5)$ & $2,385(18.5)$ & 7,842 (19.9) \\
\hline 5 (highest) & $1,005(17.7)$ & $197(14.0)$ & $3,281(16.8)$ & $2,088(16.2)$ & $6,571(16.7)$ \\
\hline \multicolumn{6}{|l|}{ CTAS score } \\
\hline 1-2 (resuscitation, emergent) & $1,425(25.2)$ & $394(27.9)$ & $6,764(34.7)$ & $1,260(9.8)$ & $9,843(25.0)$ \\
\hline 3 (urgent) & $3,176(56.0)$ & $706(50.1)$ & $9,906(50.8)$ & $5,278(40.9)$ & $19,066(48.3)$ \\
\hline 4-5 (semiurgent, nonurgent) & $1,067(18.8)$ & $310(22.0)$ & $2,815(14.5)$ & $6,361(49.3)$ & $10,553(26.7)$ \\
\hline \multicolumn{6}{|l|}{ Disposition } \\
\hline Admitted & $520(9.2)$ & $180(12.8)$ & $3,052(15.7)$ & $560(4.3)$ & 4,312 (10.9) \\
\hline Discharged & $5,148(90.8)$ & $1,230(87.2)$ & $16,433(84.3)$ & $12,339(95.7)$ & $35,150(89.1)$ \\
\hline Chest x-ray (admitted patients) & $352(67.1)$ & $140(77.8)$ & $2,263(74.5)$ & $376(67.1)$ & 3,131 (72.6) \\
\hline Chest x-ray (discharged patients) & $1,345(26.1)$ & $452(36.7)$ & 4,897 (29.8) & $2,413(19.6)$ & 9,107 (25.9) \\
\hline Overall $x$-ray rate & 1,697 (29.9) & $592(42.0)$ & $7,160(36.7)$ & $2,789(21.6)$ & $12,238(31.0)$ \\
\hline
\end{tabular}

2 years in Ontario and would likely also reduce radiograph use in those children admitted to hospital. More importantly, however, if all EDs could meet the benchmark of the lowest radiograph use by EDs with a high volume of children, 5,500 fewer children would receive a radiograph over a 2 year period for which there is no benefit and potential harm.

Common strategies across all ED types could also reduce radiograph use. Clinical pathways, standard order sets, and other clinical decision tools to bring guideline care into practice have been shown to improve inpatient care for bronchiolitis ${ }^{15,26}$ and asthma, ${ }^{18,27,28}$ as well as ED care for $\operatorname{croup}^{29,30}$ and asthma. ${ }^{18,31}$ Provider education on indications for radiographs in asthma ${ }^{32}$ and the use of a clinical practice guideline worksheet $^{33}$ show promise in reducing radiograph rates in asthma. Our findings of almost nonexistent use of clinical practice guidelines for ED care of bronchiolitis suggest that Ontario has a large gap in the implementation of these tools for common childhood conditions. However, we also found no association of current clinical practice guideline use in croup and asthma and radiograph rates. This lack of effect may relate to a lack of focus on radiograph use in the guideline's current form, as well as most guidelines not being formatted as "force functions," which have been found to improve uptake and the effectiveness of 


\begin{tabular}{|c|c|c|c|}
\hline \multirow{2}{*}{ Characteristic } & \multicolumn{3}{|c|}{ Adjusted odds ratio (95\% confidence interval)* } \\
\hline & Bronchiolitis & Croup & Asthma $^{\dagger}$ \\
\hline Male & $1.02(0.91-1.14)$ & $0.96(0.83-1.11)$ & $0.96(0.90-1.02)$ \\
\hline \multicolumn{4}{|l|}{ Age } \\
\hline 3-12 mo & 1.00 (reference) & 1.00 (reference) & $\mathrm{N} / \mathrm{A}$ \\
\hline $12-24 \mathrm{mo}$ & $1.21(1.07-1.37)$ & $1.01(0.89-1.14)$ & N/A \\
\hline $1-4 \mathrm{yr}$ & N/A & N/A & 1.00 (reference) \\
\hline $5-9$ yr & N/A & $\mathrm{N} / \mathrm{A}$ & $0.86(0.78-0.94)$ \\
\hline $10-14 \mathrm{yr}$ & N/A & N/A & $0.82(0.75-0.90)$ \\
\hline $15-19$ yr & N/A & N/A & $0.89(0.81-0.98)$ \\
\hline \multicolumn{4}{|l|}{ CTAS score } \\
\hline $1-2$ & $2.61(2.07-3.30)$ & $2.75(2.15-3.52)$ & $2.65(2.35-3.00)$ \\
\hline 3 & $1.76(1.48-2.09)$ & $1.52(1.21-1.90)$ & $1.65(1.49-1.82)$ \\
\hline $4-5$ & 1.00 (ref) & 1.00 (reference) & 1.00 (reference) \\
\hline \multicolumn{4}{|l|}{ Income quintile } \\
\hline 1 (lowest) & N/A & 1.00 (reference) & N/A \\
\hline 2 & N/A & $0.98(0.80-1.20)$ & N/A \\
\hline 3 & $\mathrm{~N} / \mathrm{A}$ & $0.75(0.61-0.94)$ & $\mathrm{N} / \mathrm{A}$ \\
\hline 4 & $\mathrm{~N} / \mathrm{A}$ & $0.90(0.75-1.09)$ & $\mathrm{N} / \mathrm{A}$ \\
\hline 5 (highest) & $\mathrm{N} / \mathrm{A}$ & $0.73(0.59-0.89)$ & $\mathrm{N} / \mathrm{A}$ \\
\hline Missing & N/A & $0.86(0.36-2.08)$ & N/A \\
\hline History of asthma & N/A & $N / A$ & $0.53(0.50-0.56)$ \\
\hline \multicolumn{4}{|l|}{ Hospital type } \\
\hline Front-line pediatric care & $0.47(0.24-0.95)$ & $0.47(0.27-0.82)$ & $0.13(0.02-0.62)$ \\
\hline Other academic & $1.24(0.83-1.84)$ & $1.47(0.86-2.52)$ & $1.78(1.23-2.56)$ \\
\hline Community with pediatricians & 1.00 (reference) & 1.00 (reference) & 1.00 (reference) \\
\hline Community without pediatricians & $0.74(0.56-0.96)$ & $0.73(0.55-0.97)$ & $0.81(0.59-1.12)$ \\
\hline
\end{tabular}

guideline care. ${ }^{34}$ Our finding that ED asthma order sets were not associated with reduced radiograph use illustrates challenges in implementing strategies in this area. Such order sets typically lay out every option for care (including radiographs), and most have no parameters for use. Pathways or more explicit criteria for radiograph use would need to be incorporated in such tools to change practice patterns. For asthma specifically, our finding that children with no previous history of wheezing are more likely to receive a radiograph in the absence of any evidence to support this practice ${ }^{35}$ speaks to the need for provider education and explicit evidencebased parameters for use. Therefore, although a mix of staffing models across EDs is likely to continue to exist for structural reasons, all institutions can implement strategies aimed at rationalizing the use of radiographs. Our findings suggest that refining the use of clinical care pathways (e.g., making the explicit omission of a radiograph the default order, instituting watchful waiting for children treated with steroids to allow time for a clinical effect or improvement prior to ordering radiographs), reinforcement through education and auditing, and an exploration of the interaction between front-line and consulting staff are three routes to optimize the use of radiographs for these common pediatric conditions.

Our study has a number of limitations. The survey data were reported by a single respondent at each hospital. Although these had been previously selected by the institution as being the most appropriate respondent, we did not verify the data provided. We did not capture the diversity of staff training (e.g., family physicians with or without additional emergency medicine training) in community hospitals. As most of our analyses were not at the provider level, we did not account for this variability but instead focused on the availability of pediatric providers, either front-line 
or in consultation. Some criteria for radiograph use relate to factors on the history or physical examination, such as a history consistent with foreign body aspiration, signs of pneumothorax in asthma, or drooling or other symptoms suggestive of obstruction in croup. Our lack of clinical data precluded an assessment of these indications or the use of standard severity risk adjustment tools for pediatric emergency patients. ${ }^{36} \mathrm{We}$ based our analyses on the final discharge diagnosis rather than the presenting complaint. Many of our study population would have presented with signs of undifferentiated respiratory distress. Although there is little evidence to suggest that radiographs are required to manage associated underlying causes (reactive airway disease, bronchiolitis), we appreciate that diagnostic imaging may be clinically indicated to rule out problems such as pneumothorax. We restricted our examinations to less ill patients (those discharged home) and attempted to control for acuity by triage scoring.

\section{CONCLUSION}

High rates of discretionary radiograph use exist for common respiratory problems of children seen across ED settings. Quality improvement efforts should be focused in this area, and radiograph use in EDs staffed by frontline pediatrics-trained staff could serve as an initial benchmark target for other institutions. Quality improvement strategies would need to include an assessment of the interaction between front-line ED staff and pediatric consultants and specifically focus on decreasing routine radiograph use when using tools such as order sets and pathways.

Competing interests: This project was funded by the Ontario Ministry of Health and Long-Term Care (MOHLTC). The funder had no role in the design or conduct of the study. Astrid Guttmann was funded by a Canadian Institutes of Health Research Career Award and Peter Austin by a Career Investigator Award from the Heart and Stroke Foundation of Ontario. This research was made possible with support from the Institute for Clinical Evaluative Sciences (ICES), a nonprofit organization funded by the Ontario MOHLTC. No endorsement by ICES or the Ontario MOHLTC is intended or should be inferred.

\section{REFERENCES}

1. Athey J, Dean JM, Ball J, et al. Ability of hospitals to care for pediatric emergency patients. Pediatr Emerg Care 2001;17: 170-4, doi:10.1097/00006565-200106000-00005.
2. Middleton KR, Burt CW. Availability of pediatric services and equipment in emergency departments: United States, 2002-03. Adv Data 2006;(367):1-16.

3. Cimpello LB, Khine H, Avner JR. Practice patterns of pediatric versus general emergency physicians for pain management of fractures in pediatric patients. Pediatr Emerg Care 2004;20:228-32, doi:10.1097/01.pec.0000121242. 99242.e0.

4. Dharmar M, Marcin JP, Romano PS, et al. Quality of care of children in the emergency department: association with hospital setting and physician training. 7 Pediatr 2008;153: 783-9, doi:10.1016/j.jpeds.2008.05.025.

5. Belfer RA, Gittelman MA, Muniz AE. Management of febrile infants and children by pediatric emergency medicine and emergency medicine: comparison with practice guidelines. Pediatr Emerg Care 2001;17:83-7, doi:10.1097/ 00006565-200104000-00001.

6. Schweich PJ, Smith KM, Dowd MD, Walkley EI. Pediatric emergency medicine practice patterns: a comparison of pediatric and general emergency physicians. Pediatr Emerg Care 1998;14:89-94, doi:10.1097/00006565-19980400000001.

7. Seeman N, Magistretti A, Baker GR. Hospital report 2005: Emergency department care. Hospital Report Research Collaborative, University of Toronto. Toronto: Joint Initiative of the Ontario Hospital Association and the Government of Ontario; 2005.

8. Pitts SR, Niska RW, Xu J, Burt CW. National Hospital Ambulatory Medical Care Survey: 2006 emergency department summary. Natl Health Stat Rep 2008;(7):1-38.

9. Guttmann A, Razzaq A, Lindsay P, et al. Development of measures of the quality of emergency department care for children using a structured panel process. Pediatrics 2006; 118:114-23, doi:10.1542/peds.2005-3029.

10. Swingler GH, Hussey GD, Zwarenstein M. Randomised controlled trial of clinical outcome after chest radiograph in ambulatory acute lower-respiratory infection in children. Lancet 1998;351:404-8, doi:10.1016/S0140-6736(97)07013-X.

11. Roback MG, Dreitlein DA. Chest radiograph in the evaluation of first time wheezing episodes: review of current clinical practice and efficacy. Pediatr Emerg Care 1998;14: 181-4, doi:10.1097/00006565-199806000-00001.

12. Ismail Y, Loo CS, Zahary MK. The value of routine chest radiographs in acute asthma admissions. Singapore Med 7 1994;35:171-2.

13. Schuh S, Lalani A, Allen U, et al. Evaluation of the utility of radiography in acute bronchiolitis. 7 Pediatr 2007;150:42933, doi:10.1016/j.jpeds.2007.01.005.

14. Christakis DA, Cowan CA, Garrison MM, et al. Variation in inpatient diagnostic testing and management of bronchiolitis. Pediatrics 2005;115:878-84, doi:10.1542/peds.2004-1299.

15. Wilson SD, Dahl BB, Wells RD. An evidence-based clinical pathway for bronchiolitis safely reduces antibiotic overuse. Am 7 Med Qual 2002;17:195-9, doi:10.1177/ 106286060201700507.

16. Knapp JF, Simon SD, Sharma V. Quality of care for common pediatric respiratory illnesses in United States emergency departments: analysis of 2005 National Hospital Ambulatory Medical Care Survey Data. Pediatrics 2008;122: 1165-70, doi:10.1542/peds.2007-3237. 
17. Canadian Institute for Health Information. CIHI data quality study of Ontario emergency department visits for fiscal year 20042005. Ottawa: Canadian Institute for Health Information; 2008.

18. Guttmann A, Zagorski B, Austin PC, et al. Effectiveness of emergency department asthma management strategies on return visits in children: a population-based study. Pediatrics 2007;120:e1402-10, doi:10.1542/peds.2007-0168.

19. Wagg J, Economou V, Razzaq A, et al. Hospital report 2007: emergency department care technical summary clinical utilization \& outcomes. Government of Ontario in partnership with the Ontario Hospital Association. Available at: http://www. hospitalreport.ca/downloads/2007/EDC/ed_report_2007.pdf (accessed November 30, 2011).

20. To T, Dell S, Dick PT, et al. Case verification of children with asthma in Ontario. Pediatr Allergy Immunol 2006; 17(1):69-76, doi:10.1111/j.1399-3038.2005.00346.x.

21. Manos D, Petrie DA, Beveridge RC, et al. Inter-observer agreement using the Canadian Emergency Department Triage and Acuity Scale. CFEM 2002;4:16-22.

22. Stenstrom R, Grafstein EJ, Innes GD, Christenson JM. The predictive validity of the Canadian Triage and Acuity Scale (CTAS). Acad Emerg Med 2009;10:512, doi:10.1197/ aemj.10.5.512.

23. Wilkins R. PCCF+ version $3 G$ user's guide (Geocodes/PCCF). Automated geographic coding based on Statistics Canada postal code conversion files, including postal codes to Fune 2001. Ottawa: Health Analysis and Modeling Group, Social and Economic Studies Division, Statistics Canada; 2001. Report No.: Cat. No. 82F0086-XDB.

24. Zeger SL, Liang KY. Longitudinal data analysis for discrete and continuous outcomes. Biometrics 1986;42:121-30, doi: $10.2307 / 2531248$.

25. Hampers LC, Faries SG. Practice variation in the emergency management of croup. Pediatrics 2002;109:505-8, doi: 10.1542/peds.109.3.505.

26. Perlstein PH, Kotagal UR, Schoettker PJ, et al. Sustaining the implementation of an evidence-based guideline for bronchiolitis. Arch Pediatr Adolesc Med 2000;154:1001-7.
27. Bailey R, Weingarten S, Lewis M, Mohsenifar Z. Impact of clinical pathways and practice guidelines on the management of acute exacerbations of bronchial asthma. Chest 1998;113: 28-33, doi:10.1378/chest.113.1.28.

28. Norton SP, Pusic MV, Taha F, et al. Effect of a clinical pathway on the hospitalisation rates of children with asthma: a prospective study. Arch Dis Child 2007;92:60-6, doi: 10.1136/adc.2006.097287.

29. Browne GJ, Giles H, McCaskill ME, et al. The benefits of using clinical pathways for managing acute paediatric illness in an emergency department. 7 Qual Clin Pract 2001;21:50-5, doi:10.1046/j.1440-1762.2001.00405.x.

30. Chin R, Browne GJ, Lam LT, et al. Effectiveness of a croup clinical pathway in the management of children with croup presenting to an emergency department. 7 Paediatr Child Health 2002;38:382-7, doi:10.1046/j.1440-1754.2002. 00011.x.

31. Gentile NT, Ufberg J, Barnum M, et al. Guidelines reduce $\mathrm{x}$-ray and blood gas utilization in acute asthma. Am 7 Emerg Med 2003;21:451-3, doi:10.1016/S0735-6757(03) 00165-7.

32. Buckmaster A, Boon R. Reduce the rads: a quality assurance project on reducing unnecessary chest $\mathrm{X}$-rays in children with asthma. I Paediatr Child Health 2005;41:107-11, doi:10.1111/j.1440-1754.2005.00559.x.

33. Gildenhuys J, Lee M, Isbister GK. Does implementation of a paediatric asthma clinical practice guideline worksheet change clinical practice? Int 7 Emerg Med 2009;2:33-9, doi:10.1007/s12245-008-0063-x.

34. Grol R, Grimshaw J. From best evidence to best practice: effective implementation of change in patients' care. Lancet 2003;362:1225-30, doi:10.1016/S0140-6736(03)14546-1.

35. Hederos CA, Janson S, Andersson H, Hedlin G. Chest x-ray investigation in newly discovered asthma. Pediatr Allergy Immunol 2004:15:163-5, doi:10.1046/j.1399-3038.2003.00098.x.

36. Chamberlain JM, Patel KM, Pollack MM. The Pediatric Risk of Hospital Admission score: a second-generation severity-of-illness score for pediatric emergency patients. Pediatrics 2005;115:388-95, doi:10.1542/peds.2004-0586. 